\title{
El Papel de los Recursos Humanos en los Nuevos Escenarios DE las ORganizaCiOnES
}

\author{
Andrés Rodriguez Fernändez \\ Victoria Zarco Martín \\ Universidad de Granada
}

RESUMEN:

Las uiltimas décadas ban estado marcadas por numerosos y profundos cambios (creciente complejidad de la economia. interdependencia de los mercados, altos niveles de competitividad, incorporación de nuevas tecnologias. etc. I que han afectado al mundo del trabajo y de las organizaciones. Son estas transformaciones las que paulatinamente han obligado a las organizaciones a establecer estrategias de actucacion claras y bien definidas, y a considerar este proceso de planificación estratégica como una berramienta imprescindible para conseguir un ajuste continuado de sus actuaciones a las demandas cambiantes del entorno.

En este marco de actuación, el presente articulo es una reflexión sobre el nuevo papel que adquieren los recursos bumanos. pues. si bien. tradicionalmente se ba entendido y aceptado quééstos desempeñaban en las organizaciones un papel subordinado y "a posteriori". en la actualidad las personas se convierten en un factor consustancial con la propia estrategia de la empresa y en un elemento indispensable en el proceso de ajuste de las organizaciones, no solo con su ambiente externo, sino también internamente.

Más concretamente, se pretende profundizar y reflexionar sobre la necesidad que las organizaciones actuales tienen de asumir como principio fundamental que el factor humano es el recurso mäs prectado que se tiene; un recurso que, asimismo, se puede desarrollar, formar e, incluso, gestionar, debiendo constituir, portanto, una de las variables estratégicas fundamentales en el proyecto de futuro de cualquier organización.

Palabras clave: cambio en las organizaciones, plan estratégico, dirección y gestión estratégica de recursos bumanos, psicología y dirección y gestión estratégica de recursos bumanos. 
ABSTRACT:
The uorld of the work and of the organizations bave been affected by numerous and deep changes occurring during the last decades (grouing complexity of the economy. interdependence of the markets. bigh levels of competitiveness, incorporation of new technologies, etc.). Due to these trans- formations the organizations gradually bave been forced to establish clear and very defined perfo- mance strategies, and to consider this process of strategic planning and indispensable tool to get a continuous adjustment from their perfomances to the changing demands of the environment. Witbin this context, the present article is considering what the new roll playing by the buman resources, because, although. traditionally it bas been understood and accepted that these ca- mied out in the organizations a subordinate paper and "a posteriori". people become a determi- nant factor at the present time. They develop and should be integrated in the oun strategic of the company and they are an indispensable element in the adjustment process of the organizations, not only with its external environment, but also internally.
Specifically, we pursue to deepen about the necessity of assuming as a fundamental principle that the buman factor is the most valuable resource currently in organizations. They are a resource that, also, can be developed, trained and, even, managed but in any case, sbould constitute, one of the critical strategic variables in the future project of any organization.

Key word: Organizational change. strategic plan, strategic management of buman resources, Psychology and strategic management of buman resources.

1. BREVE REFLEXIÓN ACERCA DE LA EVOLUCiÓN EN LA DIRECCIÓN Y GESTIÓN DE LOS RECURSOS hUMANOS

La evolución de la dirección y gestión de los recursos humanos en el contexto de las organizaciones ha sido lenta, pero gradual y progresiva, y ha estado asociada de forma estrecha a los procesos de industrialización, desarrollo económico y democrático de los países, por lo que no es de extrañar que en cada uno de ellos se haya seguido un ritmo distinto (Porret, 1997). Más concretamente, en el proceso evolutivo de la gestión del personal en las organizaciones se podrían identificar tres factores que, desde su origen, la han determinado: el entorno jurídico-social-político, tecnológico y sindical, el progresivo grado de complejidad e interdependencia de las organizaciones y la evolución de los métodos de gestión y de las ciencias humanas (Drucker, 1992, 1993).

No obstante, resulta difícil precisar dónde y cuándo tuvo lugar el origen de la aplicación de las políticas de personal, pues el sentido común nos sugiere que éstas han debido estar presentes, de un modo $u$ otro, en todas las civilizaciones que han constituido la historia humana; es decir, estamos convencidos de que en todas ellas se han aplicado técnicas sistemáticas para canalizar, estimular y dirigir el comportamiento de las personas, individual y colectivamente, a fin de alcanzar resultados más satisfactorios en los distintos contextos del trabajo. En este marco evolutivo, sin embargo, no será hasta principios del siglo XX cuando se comience a elaborar y a aplicar el conocimiento científico en este ámbito y a ello contribuyeron diversos fenómenos que, de forma escalonada, se venían desarrollando desde los dos siglos anteriores. 
Más específicamente, es a partir de la segunda crisis del petróleo cuando la dirección y gestión de los recursos humanos ha visto crecer su importancia en las organizaciones, elevándola, gradualmente, a la categoría de "estratégica" (Armstrong, 1991). Este calificativo se aplica para expresar que este campo se ha convertido en una de las principales preocupaciones de la alta dirección, orientada actualmente a desarrollar acciones de cambio, con el fin de reforzar la eficacia y los resultados de la organización en su conjunto. Igualmente, el término se utiliza para significar que actualmente en la gestión de los recursos humanos se ven implicados una gran diversidad de actores, pues, sin duda, ésta afecta y es compartida por todos los centros de autoridad en la organización hasta llegar a los responsables directos de los aspectos operativos de dicha gestión (Horwitz, 1990).

Así pues, se observa cómo la creciente complejidad de la economía, la interdependencia de los mercados, la progresiva unificación de bloques continentales y la fuerte competitividad existente, hacen que paulatinamente el proceso de análisis estratégico se configure como una herramienta imprescindible para conseguir un continuado ajuste de las organizaciones a su entorno; ajuste que, de otro lado, se considera totalmente necesario para poder adoptar en este contexto organizativo una perspectiva hacia el medio y largo plazo. Es precisamente en este marco donde los recursos humanos empiezan a convertirse en un factor determinante de la posición que la organización puede alcanzar realmente en el mercado y en un elemento diferenciador, debido a su incidencia en aspectos cruciales del quehacer de una organización, entre los cuales podemos incluir la influencia del trabajador en la mayor o menor capacidad innovadora que la empresa consiga en la gestión o en la calidad de los servicios que presta.

Este nuevo y reforzado papel que adquieren los recursos humanos en las organizaciones orienta a éstas hacia nuevas formas de hacer las cosas y hacia un cambio en su filosofía, donde ya se empieza a identificar la presencia de un subsistema psicosocial, formado por el conjunto de individuos que las componen, y, por lo tanto, a considerar la existencia en su seno de una función social. Dicha función social, obvio es decirlo, ha pasado por diversas etapas y consideraciones, las cuales explican y justifican, de algún modo, la gestión de recursos humanos que se ha llevado a cabo hasta el momento actual.

Una forma plausible, a nuestro juicio, de explicar los diversos modos de dirigir, estimular y canalizar el comportamiento humano a lo largo de la historia, es conociendo con profundidad las distintas concepciones que se han tenido acerca de la naturaleza humana y de su significado, pues dependiendo de cuáles sean éstas, así será la política de recursos humanos que se aplique y el modelo general del que se parta en las organizaciones (Benson, 1977; Zey Ferrell y Aiken, 1981). En este sentido, según Schein (1978), podemos identificar, desde un punto de vista cronológico, cuatro tipos de concepciones: 
- Concepción del hombre racional-económico. El supuesto de que se parte en esta perspectiva es que al ser humano no le gusta trabajar, y si lo hace es sólo por dinero. La teoría de la Dirección Científica del Trabajo de Taylor sería un claro exponente de esta concepción.

- Concepción basada en el hombre social. Algunos estudios, como el que llevó a cabo E. Mayo en Hawthorne, ponen de manifiesto la incidencia en la conducta de las personas de una serie de factores que no se habían considerado anteriormente, tales como la influencia de los grupos, de las relaciones interpersonales, incluso de las relaciones no formalizadas o establecidas por la organización. En este contexto, la Escuela de Relaciones Humanas sería el ejemplo más ilustrativo.

- Concepción basada en el hombre que se autorrealiza. Los principios en los que se fundamenta implican reconocer la necesidad que tienen las personas de usar y de desarrollar de forma progresiva sus propias capacidades. La teoría humanista de Maslow constituye un buen ejemplo de esta asunción.

- Concepción basada en el hombre complejo. Desde este enfoque, se reconoce la importancia, pero también la parcialidad de las formulaciones anteriores, entendiéndose que ninguna de ellas, por sí sola, es capaz de explicar el comportamiento de los individuos, $\dot{y}$ mucho menos si tenemos en cuenta las diferencias que existen entre las personas. Este aspecto, desde una perspectiva estratégica, adquiere gran importancia, ya que invita a las personas implicadas en el proceso de elaboración y de implantación de las estrategias sociales a reconocer las diferencias individuales o, al menos, las diferencias de los distintos grupos existentes en la organización. En este sentido, son las teorías de contingencia las que mejor podrían ilustrar esta concepción.

Como hemos comentado anteriormente, estas diversas asunciones sobre la naturaleza del hombre pueden ayudar a explicar las diferentes formas con que se ha llevado a cabo a lo largo de la historia la gestión de las personas, ya que el lugar que se les da y el grado de reconocimiento que obtengan en una organización es un reflejo, sin duda, de la orientación de que parten sus directivos. De todos modos, no hemos de olvidar que cada una de esas concepciones del ser humano han sido las que en cada momento histórico concitaron un mayor consenso o contaron con un mayor respaldo, por lo que al evaluarlas no podemos hacerlo considerando las exigencias sociales actuales, sino que hemos de partir del contexto histórico, es decir, de lo que la sociedad demandaba en cada uno de esos momentos.

De otro lado, y desde una perspectiva menos filosófica o más operativa, Bosquet (1982) sintetiza en tres los estadios por los que ha pasado la gestión de los recursos humanos:

Una fase administrativa, de orientación exclusivamente productivista, donde la gestión se orienta hacia el control y el estímulo del rendimiento, desarrollándose 
así acciones disciplinarias y fórmulas salariales sobre la base de los niveles de desempeño.

Una segunda fase, denominada de gestión, en la que se empieza a considerar las necesidades sociales y psicológicas de las personas con la finalidad de buscar una mejor adaptación del hombre a la organización. Sin embargo, en esta fase sigue prevaleciendo la consideración de los recursos humanos como un coste que hay que minimizar, si bien las acciones que se emprenden tienen un carácter proactivo.

En un último estadio, aparece la denominada fase de desarrollo, orientada a conciliar las necesidades económicas de la organización con las necesidades de las personas que trabajan en ella. Desde este enfoque, se entiende que la eficiencia de la organización es cada vez más tributaria de la eficiencia de la gestión social y, por tanto, de la consideración de las personas como un elemento determinante de su desarrollo. Así pues, es en esta última etapa cuando no sólo se tiene en cuenta el carácter proactivo de las acciones que se emprenden de cara al personal, sino que también se considera al individuo como un recurso que se debe optimizar, iniciándose, así, la denominada concepción estratégica de los recursos bumanos.

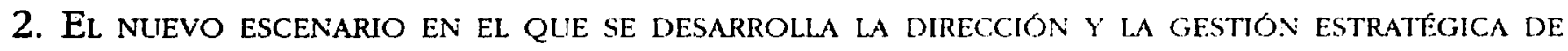
LOS RECURSOS HUMANOS

Las tres últimas décadas han estado marcadas por numerosos y profundos cambios que han incidido, como no podía ser de otra manera, en el mundo del trabajo y de las organizaciones. Así, por ejemplo, la incorporación rápida y generalizada de las nuevas tecnologías ha generado nuevas formas de trabajo, nuevos significados y nuevas formas de organización, que afectan no sólo a los sistemas de información, logística o transporte, sino también al marketing, a la gestión financiera e, incluso, a la propia producción y al empleo. Este último se sitúa en la provisionalidad o temporalidad, y se regula mediante nuevos modos de contratación vinculados a la competencia individual y a los niveles de desempeño.

Concretamente, en el contexto laboral se han desarrollado un gran número de aplicaciones tecnológicas que pueden ser agrupadas en dos grandes categorías. En la primera, estarian ubicadas todas aquéllas mediante las que se intenta subrayar la importancia del procesamiento de información a través de la informática. En la segunda, se encontrarían todas aquellas aplicaciones derivadas de las nuevas tecnologías relacionadas, por una parte, con el mundo de la oficina (ofimática o robótica) $y$, por otra, con los sistemas avanzados de producción de manufacturas, que incorpora la informática como herramienta clave tanto en el diseño como en los procesos de producción y de gestión, aproximándose así a lo que sería la factoría automatizada del futuro (Turkle, 1984; Davidow y Malone, 1992). 
Asimismo, el desarrollo de la informática y de las telecomunicaciones están haciendo posible la unificación e integración de la computadora y de la comunicación (C\&C), ya que se está produciendo una interconexión cada vez más estrecha entre ordenadores, una gran proliferación de programas, una integración progresiva entre el ordenador y el teléfono, unas grandes bases de datos integradas en el trabajo cotidiano (Bradley et al.,1993). Todos estos cambios no son sólo de carácter físico, sino sobre todo psicológico y social, pues van a afectar a los propios estilos de vida y de trabajo de las personas (Hendry, 1995).

Estas múltiples y diferentes aplicaciones tecnológicas, junto con otros factores, tales como la interdependencia de los mercados y los altos niveles de competitividad, van a tener una influencia clara sobre el diseño de estructura y sobre los procesos de trabajo que se desarrollan en las organizaciones. Veamos, de manera breve, algunas de las reestructuraciones más importantes llevadas a cabo en este contexto.

En primer lugar, en la medida en que las organizaciones crecen y se hacen más complejas, los mercados se tornan más variables y el entorno externo se vuelve más incierto, se hace necesario introducir cierto grado de flexibilidad para afrontar los cambios y las transformaciones generadas en los mercados nacionales y globales. En este sentido, cada vez es más común el concepto de producción racionalizada, desde el que se trata de combinar las nuevas técnicas de gestión con una tecnología sofisticada que permita producir más con un menor número de recursos, sobre todo humanos, o el concepto de mejora continua que implica la innovación permanente. Estos nuevos modos de concebir la gestión en las organizaciones exigen, asimismo, nuevos tipos de estructuras de carácter más descentralizado, menos jerarquizado, más flexible, constituidas por equipos de trabajo más polivalentes, más implicados en el proyecto y con un mayor grado de participación en las decisiones que se adoptan y en los resultados que se obtienen.

Por tanto, observamos que la tendencia actual consiste en desmontar las estructuras jerárquicas tradicionales, eliminando progresivamente los puestos ocupados tradicionalmente por los mandos intermedios, vertebrando y unificando diversos puestos de trabajo y un amplio número de tareas en un solo proceso y transfiriendo el control de las funciones de coordinación a los ordenadores (Laclau, 1990). Asimismo, debido a la introducción de las nuevas tecnologías, la información en la organización puede ser procesada de forma horizontal y no sólo de modo vertical, circunstancia que convierte en obsoleta la estructura piramidal tradicional, siendo más idónea una estructura de redes, donde se puede procesar un mayor volumen de información, de manera más rápida y con un menor número de personas.

Estos ejemplos no son mas que un reflejo de la tendencia existente en el mundo laboral a contemplar las nuevas tecnologías como la opción más efectiva para redu- 
cir costes y mejorar beneficios (Scott, 1999). Este proceso de automatización, incluso, se ha introducido en el ámbito de los profesionales, en la educación, en las artes, etc.; ha transgredido espacios que hasta ahora se consideraban inmunes a los avances de las tecnologías: músicos, bibliotecarios, escritores, pintores. De hecho, los procesos de reingeniería y de automatización no suponen más que el inicio de una transformación tecnológica orientada de un modo claro a incrementar y acelerar los niveles y ritmos de productividad en los próximos años. pero con una reducción drástica de personal, principalmente en las zonas bajas y medias de la estructura de la organización.

De otro lado, los cambios acaecidos en el entorno en el que se mueven las organizaciones afectan a sus pautas de legitimación, a los sistemas de valores que regulan su dinámica, a su incardinación en el sistema social, al propio concepto de trabajo, a la relación entorno interno - entorno externo, que se está ampliando casi a un nivel planetario y, finalmente, a la consideración de los elementos culturales que la componen. Así, podríamos afirmar que los tres componentes esenciales de la organización actual son la estrategia. la estructura y la cultura (Wissema, 1994) que siempre han de estar en perfecta sintonía con el entorno, con el medio exterior, por lo que cualquier acontecimiento que se produzca en ese entorno hará cambiar y redefinir, necesariamente, la composición de ese triángulo.

Por tanto, no es de extranar que para operar en un mundo lleno de incertidumbres y de cambios continuos, las organizaciones actuales deban tener estrategias claras y bien definidas, estructuras poco jerarquizadas, ágiles y susceptibles de transformarse con rapidez y culturas basadas en la excelencia y orientadas al cliente. Igualmente, en este tipo de organizaciones, el desarrollo de carreras o el itinerario profesional de los individuos es muy diferente al que se seguía en las tradicionales, pues ahora las organizaciones valoran criterios distintos de competencia que les permitan ser competitivas y sobrevivir.

Todo ello nos conduce a pensar que la movilidad en la empresa ya no va a ser lineal y vertical, sino que puede adoptar diversas formas y que las trayectorias profesionales no serán, necesariamente, verticales, sino plurales. Asimismo, la formación no va a estar orientada a la especialización sino a la polivalencia, y se valorará más el desempeño actual y el potencial del trabajador que la antigüedad. Finalmente, el desplazamiento no se considerará una disfunción, sino que se concebirá como un instrumento de desarrollo (Gifford y Pinchot, 1993).

Estos cambios en la concepción y organización del trabajo son importantes desde el punto de vista de la gestión de recursos humanos si pretendemos que el personal de ejecución se someta a reconstrucciones profesionales continuas, basadas en la polivalencia, de forma convencida. Actualmente, no se busca tan solo el incremento de fiabilidad individual, sino también colectiva a través de la adquisi- 
ción de saberes tecnológicos especializados y de procesos globales de socialización que permitan una mejor adaptación a las evoluciones de las características organizativas.

Es decir, está claro que el capital, la información, la tecnología y cualquier otro recurso que no sean las personas que constituyen una empresa se puede lograr con más o menos esfuerzo, pero ninguno de ellos, por separado o en conjunto, garantizan la consecución de buenos resultados. Sólo las personas, como agentes activos, consiguen el éxito a partir de la aplicación de otros medios, sólo ellas mejoran en términos de rendimiento y rentabilidad, mientras que los demás recursos se deprecian con el paso del tiempo. Por tanto, el éxito de las empresas se basa en las personas que las componen, en la relación que establezcan con el puesto que ocupan y en la vinculación o el compromiso con el proyecto empresarial.

\section{El NUTEVO SIGNIFICADO DE LOS RECURSOS hIimanos}

Como consecuencia de las transformaciones experimentadas tanto en el exterior como en el interior de las propias organizaciones, el Departamento de Recursos Humanos o Área de Personal, sufre una serie de cambios que pasamos a subrayar de manera breve:

\section{Se incrementan de forma significativa las competencias asignadas.}

Las acciones y los ámbitos de aplicación de los agentes implicados en la dirección y gestión de personal en las organizaciones actuales se dirigen no sólo a los trabajadores o empleados directos, sino también a los directivos y mandos de la organización; igualmente, se produce una ampliación en el horizonte temporal, pasando de la gestión de procesos de corto o medio plazo, a una basada en la previsión y en una planificación a más largo plazo (Cascio, 1989, 1995).

\section{Se amplia y enriquece el perfil y el rol profesional.}

En la actualidad, en el área de Recursos Humanos se busca a personas con una formación más polivalente, conocedoras de un discurso y de un lenguaje multifuncional: psicológico, social, jurídico y económico, que le permita relacionarse fácilmente con empleados, mandos y con la propia dirección. Estos nuevos gestores y directores de recursos humanos, a su vez, se ven apoyados en el desempeño de su actividad por auténticos especialistas en las diversas materias y su función, en definitiva, se orienta a coordinar a este equipo especializado y multidisciplinar. Así, en estudios recientes, tales como el informe de conclusiones del Proyecto Cranfield de 1995 (Tyson, Guest, Purcell, y Storey, 1997), se 
indica que se ha producido una disminución de especialistas en Derecho y, en cambio, un claro incremento de titulados en Ciencias empresariales y en Ciencias Sociales.

Este último hecho se explicaría por la visión más estratégica que se va adoptando en el área de recursos humanos, en contraposición con la visión jurídico-administrativa propia de las áreas de personal existentes en el pasado (Ordonez, 1995,1996. 1997). No obstante, esta tendencia es mucho más clara en otros paises europeos que en España, existiendo en aquéllos un porcentaje elevado de especialistas en recursos humanos con formación en Ciencias Sociales (Psicología Social y Sociología) (Soler y Suárez, 1993).

\section{Se alcanzan posiciones más altas en la estructura de la organización.}

Actualmente, los órganos de personal ocupan en las estructuras organizativas posiciones tan importantes como las del resto de funciones organizativas. Es más, su representatividad en el equipo de dirección es cada vez más alta y su incidencia en la dinámica y desarrollo de la organización en su conjunto es, asimismo, más visible y efectiva; es decir, el área de recursos humanos ha pasado de ser un mero instrumento de control a ser un área de desarrollo e innovación (Institute for Management Development, 1992).

\section{Se produce un cambio de denominación con importantes implicaciones.}

Desde la denominación de "Administración de Personal" hasta la de "Dirección y Gestión de Recursos Humanos" se han podido identificar diversos rótulos, reflejo de las diferentes concepciones de los recursos humanos que se han sustentado a lo largo de la historia, tales como Organización de personal, Relaciones sociales o Relaciones humanas. No obstante, es la dicotomía existente entre los términos "Dirección y Gestión de personal" y "Dirección y Gestión de Recursos Humanos" la que en la actualidad plantea cierta controversia, pues parece haber cierto interés por dicha diferenciación.

De hecho, algunos autores, como Guest (1994), defienden la existencia de diferencias entre Gestión de Personal y Gestión de Recursos Humanos en cuanto a las dimensiones de planificación, contrato psicológico, sistemas de control, relación con los empleados, estructura preferida, roles y criterios de evaluación. En este sentido, un dato interesante es el que se desprende de un estudio realizado por ESADE y la Universidad de Barcelona en el que se muestra cómo tanto las organizaciones españolas como las francesas prefieren más la denominación Gestión de Recursos Humanos, mientras que en el resto de países de Europa central y países escandinavos, mayoritariamente, prefieren el término Gestión de personal (Kirkbridge, 1994). 


\section{LA DIRECCIÓN ESTRATÉGICĀ DE LOS RECURSOS HUMANOS}

Si bien, tradicionalmente se ha entendido y aceptado que los recursos humanos en las organizaciones desempenaban un papel subordinado y "a posteriori", en la actualidad, éstos se convierten en un factor consustancial con la propia estrategia y en un elemento indispensable en el proceso de ajuste de las organizaciones, no sólo con su ambiente externo, sino también internamente (Narayanan y Nath, 1993).

En este sentido, se podría definir la estrategia de recursos humanos que se sigue en las organizaciones actuales como un modelo de decisión referente al área de personal, y que se plasma en un conjunto de acciones agrupadas bajo la denominación de "políticas y prácticas de gestión de recursos humanos". La finalidad de este proceso de toma de decisiones es la elaboración de una serie de objetivos y de metas desde los que se permita a la organización encontrar el ajuste más adecuado a los requerimientos del ambiente.

El método de análisis que, habitualmente, se adopta en la aplicación de este modelo, podemos resumirlo como sigue:

a. Partir de una orientación proactiva, adelantándose así a los acontecimientos e incertidumbres.

b. Establecer un claro diagnóstico acerca de las amenazas y de los potenciales peligros que pueden llegar del ambiente externo.

c. Formular objetivos y estrategias sociales que sean congruentes con el diagnóstico realizado.

d. Implantar las estrategias formuladas mediante el desarrollo de políticas de personal.

e. Concebir los recursos humanos como un factor determinante en los procesos de mejora continua y en la calidad de los servicios.

Así pues, la gestión debe emanar de la estrategia formulada en el nivel corporativo y depender de ella. En este sentido, la dirección estratégica de los recursos humanos debería ir encaminada a canalizar aquellas decisiones y acciones que conciernen a la dirección de los empleados de todos los niveles de una organización determinada y que están relacionadas con la ejecución de estrategias dirigidas hacia la creación y el mantenimiento de un alto grado de efectividad (Huselid, Jackson y Schuler, 1997).

La gestión de recursos humanos es, por tanto, un instrumento estratégico de las organizaciones, que las ayuda a modular el comportamiento de sus miembros para adecuarlo a la misión organizativa, a sus objetivos y a sus metas. Así, por ejemplo, a través de esa gestión se desarrollan las competencias; es decir, aquello que las personas son capaces de hacer como contribución al éxito de los objetivos de la organización. Asimismo, mediante esa gestión se estimula, motiva y desarrolla, humana y profesionalmente, a las personas. 
Bajo este enfoque, la verdadera gestión de recursos humanos debería concebirse como un factor estratégico de la organización que afecta a todos y que, por consiguiente, es responsabilidad de todos, aunque en mayor medida de los directivos, que serán quienes deban canalizar el comportamiento de sus miembros hacia la consecución de los objetivos de la organización. Asimismo, dicha gestión debería estar constituida por tres campos básicos:

- Desarrollo de competencias; es decir, de todo aquello que las personas son capaces de hacer como contribución al éxito de los objetivos de la organización.

- Motivación de las personas, a fin de que se sientan impulsadas a comprometerse individual y colectivamente con los objetivos planteados.

- Oportunidades disponibles por parte de las personas para hacer efectiva su contribución a la unidad u organismo en el que se desarrolle su actividad.

Será una adecuada intervención en estas tres áreas la que determinará el grado de éxito conseguido en este ámbito, ya que nos permitirá, por una parte. minimizar los eventuales conflictos entre los individuos y grupos de interés afectados y, por otra, ser eficientes y eficaces en la consecución de objetivos. Pero esa intervención. sin embargo, no puede restringirse sólo al departamento correspondiente de recursos humanos, sino que debe extenderse a todas y cada una de las áreas, servicios y negociados de la organización de un modo transversal, apoyados, eso sí, por los expertos en recursos humanos.

Desde esta concepción de la dirección y gestión cle recursos humanos, no cabe duda que nos hallamos ante un área esencial en cualquier tipo de organización, pues el comportamiento de las personas constituye la variable clave del éxito de las estrategias organizativas; es el trabajo humano el factor crítico para la producción de los productos o servicios que demandan los ciudadanos, sobre todo si tenemos en cuenta que dicho trabajo no está del todo regulado por las tecnologías, de tal manera que el margen de que se dispone para la evolución cualitativa individual es amplísimo.

En definitiva, son estos aspectos cuantitativos y cualitativos los que hacen que las organizaciones se conviertan en clientes especiales de la dirección y gestión de los recursos humanos. Por ello, aquéllas habrán de prestar un apoyo decidido a esta área para poder superar progresivamente los principales problemas con los que se encuentra.

5. LA DIRECCION ESTRATÉGICA DE LOS RECURSOS HUMANOS COMO DISCURSO RETÓRICO

Paradójicamente, a lo largo del proceso de modernización de las organizaciones, las políticas de reforma de los recursos humanos son las que han planteado más resistencias y donde los cambios que se han producido han sido más lentos. Una de las razones que explican esta resistencia es la propia inercia, es decir, la 
Andrés Rodriguez Fernandez - Victoria Zarco Martinez

tendencia de las organizaciōnes a mantener su funcionamiento tradicional, orientado exclusivamente hacia los resultados y no hacia los procesos, ni hacia los clientes, ni, por supuesto, hacia los propios empleados, en su calidad de clientes internos.

Incluso, aún hoy en día, las previsiones de personal no están vinculadas a un plan estratégico, ni a unos objetivos generales concretos; esto es, no existe una política activa de reclutamiento, la selección sigue estando más orientada a la lealtad y a la confianza que a la calidad del resultado y existe un excesivo número de normativas reguladoras del personal, que lleva a la uniformidad, a la rutina y a la rigidez. Estas circunstancias se agravan si tenemos en cuenta que no suele existir en las organizaciones un plan de desarrollo de carreras, estructurado y vinculado al desempeño, por lo que el desarrollo de las actividades se caracteriza por su impersonalidad y los sistemas retributivos por estar más ligados a la categoría y al puesto, que a los propios objetivos y resultados a conseguir. Finalmente, se observa habitualmente la ausencia de un plan de formación integrado en un plan estratégico previo, así como un excesivo grado de concentración de competencias en los sistemas centrales.

Además, se constata que en el modelo actual de dirección y gestión de recursos humanos no se desarrollan las competencias directivas que requiere el sistema empresarial, ni se garantizan la ubicación y el aprovechamiento más adecuado del personal. En la base de estos fallos, se encuentran la excesiva centralización de las decisiones en materia de personal, el desinterés de los directivos hacia los trabajadores, bien por considerar que tales responsabilidades no les competen, o bien por rehuir el conflicto, y el bajo desarrollo de la planificación, racionalización y reasignación de recursos humanos, con lo que se producen amplias parcelas infradotadas y otras, por el contrario, con exceso de personal. Por último, también nos encontramos con severas disfunciones en el diseño de carreras, que no se adaptan a las necesidades y expectativas actuales, la inexistencia de una relación contingente entre desempeño y sistemas de recompensas (retribución y/o promoción) y la presencia de cierta rigidez en los procedimientos, que favorece la inacción y el estancamiento.

Para superar esta situación, es necesario pasar de un discurso retórico acerca de la importancia estratégica de los recursos humanos a un discurso efectivo, desde el que se conceda a las personas el peso que realmente tienen. Sólo bajo este enfoque será posible llevar a cabo un rediseño organizativo, tendente a la descentralización y a la asunción de responsabilidades, así como incrementar realmente la capacidad organizativa para desarrollar las políticas de recursos humanos, sobre todo aquellas que introducen innovaciones o cambios. Asimismo, es esta perspectiva la que puede ayudar a actualizar el marco jurídico con el fin de adaptar los recursos humanos, no a la uniformidad, sino a la rica heterogeneidad que demanda la acción empresarial y de flexibilizar 
las plantillas, eliminando, así, una buena parte de la carga garantista que hace. a veces, tan rígida la gestión.

Sin duda, para llevar a cabo estas transformaciones se requiere el fortalecimiento de la dirección, tanto desde el punto de vista interno como externo, a fin de que ésta pueda asumir responsabilidades específicamente gerenciales, así como urge la implantación de un desarrollo de la carrera profesional, basado en la competencia, en el desempeño profesional y en el receptor del servicio. En esta situación sería plausible la externalización y privatización de los servicios, una vez formulados los objetivos, planificadas las acciones y disenados los sistemas de evaluación y de seguimiento.

Asimismo, los recursos humanos deberán ser integrados en un proyecto de organización compartido, sustentado en una estructura descentralizada y aplanada y en una cultura orientada al ciudadano, a la calidad y a la excelencia. Por último, para pasar de la concepción tradicional de "Organización de Personal" a la concepción actual de "Dirección y Gestión de Recursos Humanos" es también necesario contar con el compromiso y la implicación de los empleados, con la tecnología adecuada y con unos planes efectivos de formación integral continua y de desarrollo de carreras (González y De Elena, 1998; González, 1999).

Por tanto, frente al modelo clásico de personal, proponemos un enfoque para el análisis de la organización que se centra en la trama de relaciones y de actividades, construida sobre imágenes compartidas por los participantes, que perduran a pesar de los cambios en la tecnología e inestabilidad del entorno. Este enfoque de organización y de gestión giraría en torno a tres elementos esenciales:

a. Identificación y delimitación de unidades organizativas que estén constituidas en torno a una función esencial.

b. Carácter autónomo de dichas unidades, con la finalidad de que puedan responsabilizarse de los resultados.

c. Flexibilidad en la organización y el funcionamiento de estas unidades, tanto interno como externo, para poder afrontar los cambios de un modo satisfactorio.

6. LA DIRECCIÓN Y GESTIÓN DE RECURSOS hUMANOS COMO ELEMENTO CLAVE EN EL PLAN ESTRATÉGICO DE LAS ORGANIZACIONES ACTUALES

Una de las funciones básicas de la alta dirección es definir el plan estratégico, es decir, el proyecto de lo que se va hacer en la organización. En la elaboración de ese plan, las organizaciones actuales deberán asumir como principio fundamental que el factor humano es el recurso más preciado que se tiene y el que, asimismo, más facetas y complejidades presenta, debiendo constituir, por tanto, una de las variables estratégicas fundamentales de dicho proyecto de futuro. 
Desde este enfoque, comò hemos esbozado en el epígrafe anterior, la dirección estratégica de los recursos humanos se puede concebir como un proceso compuesto de diferentes fases: diagnóstico del ambiente externo (amenazas y oportunidades) y del ambiente interno (fuerzas y debilidades) de la organización, formulación de objetivos y de estrategias sociales que sean congruentes con el diagnóstico realizado e implantación de las estrategias formuladas mediante el desarrollo de políticas de personal. Pasemos a desarrollar brevemente las diferentes etapas de este proceso estratégico.

\section{El ambiente externo}

Desde que las organizaciones se conciben como sistemas abiertos, el análisis del entorno o ambiente en el que actúan se configura como un aspecto esencial si aquéllas quieren alcanzar unos niveles adecuados de efectividad. Este ambiente incluye los objetos, las personas y demás organizaciones que rodean a un sistema organizativo específico, además de las fuentes de insumos que utilizan las organizaciones y los clientes de los productos y servicios producidos.

Por tanto, se necesita realizar un diagnóstico de dicho ambiente para determinar las amenazas y las oportunidades que éste presenta para la organización, así como para fijar su posición de efectividad. No obstante, para ello hemos de delimitar e identificar, en primer lugar, el ambiente general o macroambiente, que estaría formado por el conjunto de condiciones que afectan a la totalidad de las organizaciones y que son potencialmente relevantes para la organización de que se trate. En segundo lugar, nos centraríamos en el ambiente específico, que se caracteriza por su gran incidencia sobre la organización focal y lo componen aquellas unidades e individuos que se relacionan directamente con nuestro sistema. Como indica el profesor Valle (1995), en cada uno de esos niveles de análisis pueden identificarse diversos componentes de distinta naturaleza.

\section{El ambiente interno}

Los principales factores o variables a tener en cuenta en el diagnóstico del ambiente interno de una organización son los siguientes:

- Misión

El concepto de misión nos remite a los cimientos del proyecto de una organización, al punto de partida de ésta, a la finalidad esencial que legitima su existencia. En este sentido, algunos autores defienden que las organizaciones existen para cumplir una misión, la cual puede ir cambiando conforme lo hace su entorno (interno y externo), así como las personas que tienen la capacidad para definirla o fijarla. En suma, la misión constituye el marco que inspira el conjunto de actuaciones de una organización. 


\section{-Cultura organizativa}

La cultura se puede definir como los sistemas de valores, creencias, normas y símbolos creados por una organización, comparticlos por la amplia mayoría de sus miembros, y que influyen en los comportamientos de los individuos, tanto desde el punto de vista personal como colectivo. Así pues, ésta se convierte en un instrumento poderoso para alcanzar las metas de la organización. al condicionar no sólo las percepciones de los trabajadores, sino también la identificación de la misión, la formulación de las estrategias a seguir y el grado de éxito que se puede lograr con su implantación.

Por ello, es necesario que la organización realice un diagnóstico y conozca su perfil cultural con la finalidad de alinear la planificación estratégica con las claves culturales existentes en la organización, teniendo en cuenta que en el proceso de formación de la cultura los recursos humanos juegan un papel fundamental, por lo que sólo mediante procesos de formación y mediante el establecimiento de programas de compensación dirigidos al personal podremos fortalecer la cultura existente o bien modificarla.

\section{-Estructura}

La estructura se relaciona con los aspectos más estables de la organización. nos ayuda a predecir lo que sucede en ella y, en cierta medida, nos posibilita controlar los procedimientos a través de los cuales se realizan sus actividades. Más concretamente, siguiendo la definición propuesta por Kast y Rosenzweig (1988), la estructura implica:

- un patrón de relaciones y de obligaciones formales (organigrama).

- la manera en que se asignan las distintas actividades a los diferentes departamentos y personas (diferenciación).

- el modo en que tales actividades se coordinan entre sí para lograr los objetivos de la organización (integración).

- la forma en que se desarrollan las relaciones de poder y de estatus en la organización (sistema de autoridad).

- los procedimientos formales que guían las actividades y las relaciones de los miembros en la organización (sistema administrativo).

Este tipo de estructura es la que, habitualmente, llamamos formal y responde a las previsiones y a la voluntad de la organización. Pero, inherente a ella, encontramos también la estructura informal, como si de la cara y cruz de una moneda se tratara, pues siempre que hablamos de la dimensión formal hemos de referirnos, necesariamente, a la dimensión informal, que surge de una manera espontánea e imprevista por la organización a fin de cubrir las múltiples lagunas que no se han podido prever con la estructura formal. Ambos tipos de estructuras configuran una realidad indisociable que es la organización. 


\section{Formulación de la estrategia social}

Una vez realizados el diagnóstico externo e interno es cuando se está en condiciones de formular los objetivos en el área social. Esta etapa de formulación de objetivos es fundamental en el plan estratégico, ya que constituye el marco de referencia a partir del cual se definirán las estrategias sociales, así como los distintos planes de acción en que éstas se concretan.

Dichos objetivos estratégicos en la gestión de los recursos humanos han de elaborarse a partir de una serie de criterios que, siguiendo a Evans (1996), podríamos resumir en los tres siguientes:

\section{Equidad, integración y participación.}

La percepción de equidad por parte de los trabajadores es fundamental si queremos que en el seno de cualquier organización se desarrollen actitudes y comportamientos funcionales, entre los cuales se encuentran el deseo de la persona de integrarse y, por ende, de participar en las actividades de la empresa, traduciéndose todo ello en un compromiso moral con respecto al logro de sus objetivos.

\section{Rendimiento operativo.}

Se pretende que las personas alcancen mediante su desempeño los resultados esperados. Ello requiere, por una parte, la fijación de unos objetivos operativos claros, factibles y estimulantes y, por otro lado, el establecimiento de un sistema de recompensas que esté ligado al rendimiento de las personas y a los resultados globales de la organización.

\section{Innovación y flexibilidad.}

Estos son dos requisitos necesarios para desenvolverse de forma eficaz en un ambiente cambiante y dinámico. A través de la innovación, se pretende aplicar sistemas de relaciones interpersonales e interdepartamentales directos, disminuyendo, así, las rigideces derivadas de las relaciones formales. La flexibilidad requiere, por su parte, variabilidad salarial, diversidad en la contratación laboral y polivalencia en la organización del trabajo.

\section{Implantación de la estrategia de recursos bumanos}

El esfuerzo analítico de las etapas anteriores nos permite la implantación de estrategias en el área de los recursos humanos, cuya articulación dentro del plan estratégico global, supone integrar los diferentes niveles organizativos implicados: directivo, táctico y operativo, mediante los cuales se instrumentalizarán las estrategias y podrán desarrollarse en políticas y acciones concretas. 
Aunque es difícil, debido al gran número de contingencias que se presentan, exponer todos los posibles factores de éxito que intervienen en la implantación de la estrategia social en el seno de una organización. a continuación sugerimos algunos de los más relevantes:

- Planificación a largo plazo y clima/cultura de apoyo. Es necesario crear un clima y una cultura de apoyo, en sincronía con una planificación a largo plazo. Esto ayudará a preparar mejor las capacidades necesarias para la consecución satisfactoria del proyecto.

- Contenidos, procedimientos y finalidad. Los empleados tendrán que saber qué hacen, cómo lo hacen y con qué finalidad lo hacen, a fin de que puedan vincularse y comprometerse con el trabajo que realizan y con el proyecto global con el que se identifican.

- Ajuste de estructuras, sistemas y procesos. La planificación estratégica requiere un ajuste global de estos tres factores y del personal afectado, si bien, nunca podemos pretender que tal ajuste sea perfecto.

- Participación y disponibilidad para asumir el cambio. Se necesita mucho tiempo de negociación para el desarrollo de las decisiones estratégicas. En tal sentido, hay que prever medios de control para detectar posibles desviaciones en los planteamientos iniciales, sujetos continuamente al cambio y a nuevas interpretaciones.

- Definición de un marco realista de referencia temporal para el cambio. Se requiere un mínimo de dos a tres años, y con frecuencia cinco, para poner en marcha procedimientos estratégicos de forma suave, máxime en las organizaciones actuales donde son tan numerosos los actores implicados y tan heterogéneos sus intereses.

- Los procesos han de concebirse como un medio orientado a un fin. La reflexión estratégica es una forma de pensar respecto de las circunstancias actuales y futuras, teniendo siempre presente cuál es la finalidad del proceso.

Sin duda, todo este proceso de gestión estratégica de los recursos humanos debería sustentarse en la asunción por parte de la organización de dos supuestos básicos: los recursos humanos son el factor crítico de éxito en las organizaciones y la gestión eficaz de éstos es el instrumento básico para el logro de sus fines. No obstante, desde un enfoque psicológico, social y crítico de los recursos humanos, creemos que nuestro objetivo fundamental como psicólogos ha de ser ayudar a las organizaciones a crear las condiciones idóneas para mejorar la vida de las personas que trabajan en ellas.

Desde esta perspectiva, por tanto, los Recursos Humanos habrian de definirse básicamente en términos de nuevas prácticas; es decir, deberíamos ofrecer modelos teóricos y herramientas para producir conocimiento emancipador y ayudar, así, a desarrollar prácticas que posibiliten otro tipo de racionalidad y modos diferentes de relación en las organizaciones. Al desarrollo de esta idea dedicamos el final de esta reflexión sobre la gestión estratégica de los recursos humanos. 
7. El papel de la Psicologta en la gestión y diRección estratégica de recursos humanos

Como acabamos de esbozar, en este nuevo marco en el que nos movemos, es necesario reflexionar sobre las aportaciones de la Psicología al crecimiento y al desarrollo personal, laboral y social de los trabajadores. En este sentido, creemos que hay que poner en entredicho que el avance de nuestra disciplina en el estudio del comportamiento humano deba seguir los requerimientos y las directrices de las organizaciones e instituciones, orientadas exclusivamente a lograr posiciones de excelencia y de liderazgo en los mercados. Por el contrario, pensamos que se debería profundizar en el estudio del comportamiento humano con la finalidad de lograr nuevas formas de relación (psicológica, social, económica, política) entre los miembros de una empresa, que les dote de mayores niveles de libertad, de emancipación, de saberse sí mismos, con identidad, con autonomía y con dominio razonable de su propio proyecto (Tsoukas, 1995).

Así, por ejemplo, en relación con las nuevas tecnologías, el problema que debiéramos plantearnos, como psicólogos del trabajo, es el modo en que éstas afectan a las destrezas requeridas, a las características del puesto o a las relaciones humanas en el contexto de trabajo, pues lo importante, desde un punto de vista psicosocial, no es la nueva tecnología que se implante, sino la forma como se implante. Hemos de pasar de un enfoque centrado en la tecnología a otro centrado en el usuario final y en la organización.

Asimismo, hay que ser conscientes de que las nuevas tecnologías están generando cambios sobre los sistemas de producción que, consecuentemente, repercutirán sobre el mercado de trabajo y sobre la sociedad en general. En este sentido, Pestel (1998) apuntaba que la mitad de los puestos de trabajo se vuelven obsoletos cada cinco años en EE.UU. Además, la automatización creciente del trabajo fomenta, a pesar de los cantos de sirena de los políticos y de los correligionarios del progreso, una situación de auténtico paro estructural (Schaff, 1985). Ambas situaciones -obsolescencia acelerada de los puestos de trabajo y paro estructural- llevan a una gran parte de la población a vivir en un contexto acelerado, tenso y plagado de ansiedad, debido a los altos costes que supone para este gran número de personas superar las barreras y dificultades, a veces dramáticas, que supone esta situación.

Este panorama, se agrava al existir profundos desajustes entre la moral tradicional del trabajo, que sitúa a éste como fundamento de la vida social, y la nueva realidad emergente, que lo relega a mero instrumento de cambio. En este sentido, podemos constatar cómo la sociedad actual, al situar todavía el trabajo y la práctica de un oficio como el fundamento de la vida social, sume a la persona carente de un empleo en un profundo trauma, pues desde el derecho a la jubilación hasta el prestigio social, y desde las oportunidades de formación hasta la propia identidad individual giran aún en torno a éste (Braverman, 1974). Por último, no cabe duda de 
que los cambios acaecidos están transformando la propia identidad del ser humano y convirtiéndola en otra identidad más consumista, acrítica e insolidaria, típica de la era de la globalización, en la que ya estamos instalados (Solé, 2000).

Este es, en definitiva, el escenario desde el que hay que considerar a las organizaciones como espacios de gobierno, en los que confluyen saberes y prácticas que ordenan y diferencian a personas y a poblaciones, produciendo economías potentes y globales, en unos casos, y eriales de violencia, hambre y miseria en otros (Brown. Flavin y French, 1999). Es, igualmente, esta nueva realidad la que nos permite redefinir los discursos tradicionales sobre las organizaciones y pasar a considerarlas actualmente como verdaderos instrumentos de control y de poder (Rodríguez, 1994: Storey, 1995).

Sin embargo, nadie se ha preocupado de evaluar los costes psicológicos y humanos que estos avances tecnológicos pueden generar $y$, de hecho, generan en las personas y en las sociedades. Parece que sólo fuese importante la eficiencia y el beneficio, cuando es realmente el ser humano quien debiera constituir el referente esencial de todo progreso y de todas las acciones que realizamos, pues cabe suponer que todo cuanto hacemos, inventamos, creamos y desarrollamos está dirigido a incrementar el bienestar y la calidad de vida de las personas, de la mayoría de las personas, de todas las personas (Rodriguez, 1992).

Bajo esta perspectiva, creemos que concebir el mundo laboral y vital del hombre como un conjunto de problemas instrumentales, resolubles por la ciencia y la técnica y orientado exclusivamente al mejoramiento material de las condiciones de vida, implica optar por un reduccionismo de consecuencias imprevisibles (Rorty, 1991). Este pragmatismo que alienta la vida del trabajo y la vida en general de las personas, sostenido por la técnica y dirigido hacia la eficiencia, ya no es ni tan siquiera funcional para el hombre, sino meramente utilitario.

Sin duda, hemos de encontrar mecanismos o cursos cle acción que nos lleven a instalarnos en otro tipo de relaciones humanas, en otro modelo de organizaciones e instituciones, en otro sistema social, político y económico (Küng, 1995). Es decir, hemos de pensar desde otro paradigma donde sea posible encontrar alternativas válidas para generar un cambio en los pilares en los que se sustenta el actual sistema (Pfeffer, 1993), y, así, poder ayudar a las personas privilegiadas del mundo occidental, que es donde estamos, a desarrollar su proyecto de vida sin tanta ansiedad, acoso, miedo, insatisfacción y estrés. Igualmente, desde este nuevo paradigma, debería ser posible la existencia de organizaciones que asuman la excelencia no sólo en su dimensión económica, sino también humana.

No obstante, en la literatura al uso, no sólo la orientada a la dirección y gestión empresarial, de profundo calado economicista, sino desde la propia Psicología del Trabajo y de las Organizaciones, se ha venido considerando a los recursos humanos en un sentido casi exclusivamente instrumental; es decir, en términos de eficiencia, de competencia, de valor, de utilidad, lo cual podría ser lógico desde una racionalidad 
económica, pero en modo àlguno desde lo que tantas veces se pregona en los órganos más reconocidos de la Psicología institucional como su razón de ser: ayudar a las personas y a los grupos a lograr mayores niveles de bienestar psicológico y social.

Así pues, el enfoque bajo el que se ha desarrollado la literatura acerca de los recursos humanos, incluso desde la Psicología, está profundamente impregnado, como es lógico, de los valores dominantes de la cultura en la que se desarrolla (Brunson, 1989). Los teóricos y profesionales tenemos una gran responsabilidad, por tanto, en la forma en que construimos la disciplina y la aplicamos a la realidad laboral y, en tal sentido, habría que hacer un esfuerzo porque los saberes desde la que se construya sean emancipadores y las prácticas sociales mediante las que se apliquen los conocimientos sean y estén orientadas al crecimiento humano.

Esto supondría un primer paso para que se produzca un giro sustantivo en lo que ha venido siendo la evolución de los recursos humanos, cuyo verdadero interés se ha puesto y se sigue poniendo en el control y en la predicción (Habermas, 1987), gestándose un sesgo epistemológico, al subrayar o colocar el énfasis en cómo deben comportarse los individuos desde el punto de vista de las organizaciones e ignorando su propia perspectiva (Foucault, 1986; Latour, 1987).

Hemos de partir del hecho de que la conducta humana es esencialmente propositiva, con un carácter intencional y, en gran medida, impredecible. Sin embargo, los teóricos, los técnicos y los responsables de los recursos humanos hemos terminado por identificarnos con la racionalidad y con la lógica de la eficiencia empresarial. Consecuentemente, hemos terminado por elaborar un modelo de hombre compatible y coherente con esa lógica y una epistemología y una metodología que únicamente nos permite avanzar en el conocimiento que ésta nos posibilita, en el conocimiento del hombre racional y eficiente dedicado a trabajar para consumir, gastar y volver a trabajar o pensar en ganar más (Du Gay, 1996).

En este sentido, creemos que es necesario, como ya indicábamos al inicio, desarrollar una teoría acerca del comportamiento humano en las organizaciones desvinculada del desarrollo de la ideología que legitima y apoya la organización y la cultura empresarial derivada del enfoque dominante Clegg y Dunkerley, 1980). Dicha teoría tendría que partir de la comprensión de los múltiples modelos existentes sobre el comportamiento humano en las organizaciones y analizar si en el contexto sociohistórico actual es posible una articulación teórica partiendo de supuestos epistemológicos diferentes. Ello implicaría realizar un desbrozamiento en la amplia gama de enfoques instrumentales que se desarrollan en esta área, analizando el modo en que el contexto en que se ha desarrollado ha influido en su evolución y tomando en consideración las concepciones de que parten los propios responsables de las organizaciones acerca la naturaleza humana y cómo desde esas concepciones se han contextualizado los problemas y se han desarrollado lás prácticas que hoy se aplican. 
Elaborar una nueva teoría sobre el comportamiento humano en las organizaciones, implica, por tanto, conocer lo que tenemos, puesto que el campo que nos ocupa es diverso y algo difuso, con la aspiración de orientarla hacia el desarrollo y crecimiento de la dimensión humana. Esto requiere plantearse. como es lógico, otro tipo de preguntas que las que se han formulado hasta ahora acerca de las organizaciones y de los recursos humanos. También exige definir con claridad la unidad de análisis que, en nuestro caso, tendría que ser el individuo y el grupo, si en verdad nuestro foco de atención va a ser los fenómenos y procesos psicológicos y sociales que se desarrollan en el contexto de las organizaciones, pues la perspectiva que adoptemos sobre la acción cle las personas y la unidad de análisis que utilicemos varía según que interese comprender los efectos que las organizaciones tienen en la sociedad, los que tienen en los individuos - bien la manera de manejar y controlar a las personas que trabajan en ellas (Pfeffer, 1982, 1993).

\section{Bibliografía}

Armstrong, M., (1991). A Handbook of Personnel Mana-gement Practice. London: Kogan Page.

Benson, J.K., (1977). Organizational Analysis: Critique and Innovation. Beverly Hill CA: Sage.

Bradley, St., et al., (1993). Globalization technology', and competition: The fusion of computers and telecommunications in the 1990s. Cambridge, MA: Harvard Business School Press.

Braverman, H., (1974). Labor and monopoly capital. New York: Mountly Review Press. Brown, L., Flavin, C. y French, H., (1999). La situación del mundo. Informe anual del Worldwatch Institute. Madrid: Icaria.

Brunson, N., (1989). The organization of bipocrisy: Talk, decisions and actions in organizations. Chichester: Wiley.

Cascio, W., (1989). Managing buman resources: producti-vity. quality of work life,profits. New York: McGraw- Hill.

Cascio, W., (1995). "Wither industrial and organizational psychology in changing world of work". American Psychologist, 50/11, 928-939.

Clegg, St. y Dunkerley, D., (1980). Organization, Class and Control. Londres: Routledge and Kegan Paul.

Davidow, W. y Malone, M., (1992). The virtual corporation: structuring and revitalizing the corporation for the 21st century. New York: Harper Collins.

Drucker, P., (1992). "The new society of organizations". Harvard Business Review, Spt-Oct, pp 95-104. 
Drucker, P., (1993). La sociedad postcapitalista. Barcelona: Apóstrofe.

Du Gay, P., (1996). Consumption and identity at work. London: Sage.

Evans, P., (1996). Dirección internacional de recursos bumanos. Técnicas y retos.

Congreso Mundial de Dirección de Personal, Hong Kong, 25-28 de Junio.

Foucault, M., (1986). "Space, knowledge and power". In Rabinow, P (ed): The Foucault reader. Harmondsworth: Penguin.

Gifford, R. y Pinchot, E., (1993). The end of bureaucracy and the rise of the intelligent organization. San Francisco: Berrett-Koehler Publishers.

González L., y De Elena J., (1998). "Propuesta de un modelo de dirección y gestión de recursos humanos". Revista de Trabajo y Seguridad Social, 189, 95-150.

González, L., (1999). "Modelos de dirección de recursos humanos". Papeles del Psicólogo, 72, 25-34.

Guest, D., (1994). Organizational psychology and human resources management: Towards a european approach. European Journal of Work and Organizational Psychology, 4, 3,251-270.

Habermas, J., (1988). Teoría de la acción comunicativa. Madrid: Taurus.

Hendry, C., (1995). Human resource management: A strategic approach to employment. London: Butterworth.

Horwitz, F., (1990). "HRM: An ideological perspective". Personnel Review, 19 (2) $10-$ 15.

Huselid, M., Jackson, S. y Schuler, R., (1997). "Technical and strategic human resource management effectiveness as determinants of firm performance". Academy Management Journal, 40,1,171-188.

Institute for Management Development, 1992. The emergent role of the resources buman manager in Europe. Lausana, EAPM.

Kast, F. y Rosenzweig, J., (1988). Administración en las organizaciones: enfoque de sistemas y de contingencias. México: McGraw-Hill.

Kirkbridge, P (ed)., (1994). Human resource management in Europe. London: Routledge.

Küng, H., (1995). Proyecto de una ética mundial. Madrid: Trotta.

Laclau, E., (1990). New reflections on the revolution od our time. London: Verso.

Latour, B., (1987). Science in action. Milton Keynes: Open University Press.

Narayanan, V y Nath, R., 1993. Organization theory. A strategic approach. Homewood, Irwin.

Ordóñez, M, (1995). La nueva gestión de los recursos bumanos. Barcélona: Gestión 2000.

Ordóñez, M, (1996). Modelos y experiencias innovadoras en la gestión de los recursos bumanos. Barcelona: Gestión 2000. 
Ordóñez, M, (1997). Psicologia del trabajo y gestión de recursos bumanos. Barcelona: Gestión $2000 /$ Aedipe.

Pestel, E., (1998). Cambios, retos y experiencias en la transformación de la sociedad informática. Valencia: Presidencia de la Generalitat.

Pfeffer, J., (1982). Organizations and organization theory, Manshfield, M.A.: Pitman. Trad. castellano. B. Aires. El Ateneo. 1987.

Pfeffer, J., (1993). "Barriers to the advance of organizational science: paradigm development as a dependent variable". Academy of Management Rev'iew, 18 (4) 599-620.

Porret, M., (1997). "Evolución histórica y situación actual de la Dirección y Gestión de los Recursos Humanos en las organizaciones". Revista de Trabajo y Seguridad Social. 171, 125-176.

Rodríguez, A. (1992). Psicología de las organizaciones. Teoría y' método. Barcelona: PPU.

Rodríguez, A. (ed.) (1994). Las organizaciones a debate. Granada: Servicio de Publicaciones de la Universidad de Granada.

Rorty, R., (1991). Objectivity, relationism and trutb. Cambridge: Cambridge University Press.

Schaff, A., (1985). ¿Qué futuro nos aguarda? Barcelona: Crítica.

Schein, E., (1978). Psicología de la organización. Madrid: Prentice-Hall International.

Scott, M., (1999). 'El futuro será de quienes creen valor. Bilbao: Deusto.

Solé, M., (2000). Los consumidores del siglo XXI. Madrid: Esic.

Soler, J y Suarez, G., (1993). "La gestión de recursos humanos en Europa: ¿Hacia dónde vamos?". Capital Humano, 59. 12-18.

Storey, J., (ed)., (1995). Human resource management: A critical test. London: Thompson International.

Tsoukas, H., (1995). The ubiquity of organizational diversity: A social constructivist perspective. University of Warwick: Warwick Business School.

Turkle, S., (1984). The second self: Computers and the buman spirit. New York: Simon \& Schuster.

Tyson, S., Guest, D., Purcell, J. y Storey, J. (1997). Report to ESRC on The link between HRM and business performance. Cransfield: Cransfield School of Management.

Valle, R., (1995). Gestión de Recursos Humanos. Madrid: Addison Wesley.

Wissema, J., (1994). Dirección de empresas descentralizadas. Barcelona: Folio. Zey-Ferrell, M. y Aiken, M. (eds.). (1981). Complex organizations: Critical perspectives. Glenview Ill: Scott Foresman. 